\title{
Participation of liver progenitor cells in liver regeneration: lack of evidence in the $\mathrm{AAF} / \mathrm{PH}$ rat model
}

\author{
Ange-Clarisse Dusabineza ${ }^{1}$, Noémi K Van Hul ${ }^{1}$, Jorge Abarca-Quinones ${ }^{1}$, Peter Starkel ${ }^{1}$, Mustapha Najimi ${ }^{2}$ and \\ Isabelle A Leclercq ${ }^{1}$
}

When hepatocyte proliferation is impaired, liver progenitor cells (LPC) are activated to participate in liver regeneration. We used the 2-acetaminofluorene/partial hepatectomy (AAF/PH) model to evaluate the contribution of LPC to liver cell replacement and function restoration. Fischer rats subjected to AAF/PH (or PH alone) were investigated 7, 10 and 14 days post-hepatectomy. Liver mass recovery (LMR) was estimated, and the liver mass to body weight ratio calculated. We used serum albumin and bilirubin levels, and liver albumin mRNA levels to assess the liver function. LPC expansion was analyzed by cytokeratin 19 (CK19), glutathione S-transferase protein (GSTp) immunohistochemistry and by CK19, CD133, transforming growth factor- $\beta 1$ and hepatocyte growth factor mRNA expression in livers. Cell proliferation was evaluated by Ki67 and BrdU immunostaining. Compared with PH alone where LMR was $~ 100 \% 14$ days post-PH, LMR was defective in AAF/PH rats $(64.1 \pm 15.5 \%, P=0.0004)$. LPC expansion was scarce in PH livers $\left(0.5 \pm 0.4 \%\right.$ of $\mathrm{CK} 19^{+}$area), but significant in AAF/PH livers $\left(8.5 \pm 7.2 \%\right.$ of $\left.\mathrm{CK}_{1} 9^{+}\right)$, and inversely correlated to $\mathrm{LMR}\left(r^{2}=0.63, P<0.0001\right)$. A quarter of $\mathrm{AAF} / \mathrm{PH}$ animals presented liver failure (low serum albumin and high serum bilirubin) 14 days post- $\mathrm{PH}$. Compared with animals with preserved function, this was associated with a lower LMR ( $50 \pm 6.8$ vs $74.6 \pm 9.4 \%, P=0.0005)$, a decreased liver to body weight ratio $(2 \pm 0.3$ vs $3.5 \pm 0.6 \%, P=0.001)$, and a larger LPC expansion such as proliferating Ki67 ${ }^{+}$LPC covered $17.4 \pm 4.2 \%$ of the liver parenchyma vs $3.1 \pm 1.5 \%$, $(P<0.0001)$. Amongst those, rare LPC with an intermediate hepatocyte-like phenotype were seen. Also, less than $2 \%$ of hepatocytes were engaged into the cell cycle $\left(\mathrm{Ki}_{6} 7^{+}\right)$, while more numerous ( $\sim 25 \%$ of hepatocytes) in the livers with preserved function. These observations suggest that, in this model, the efficient recovery of the liver function was ensured rather by the proliferation of mature hepatocytes than by the LPC expansion and differentiation into hepatocytes.

Laboratory Investigation (2012) 92, 72-81; doi:10.1038/labinvest.2011.136; published online 12 September 2011

KEYWORDS: 2-acetaminofluorene; liver function; liver progenitor cells; partial hepatectomy; regeneration

In the healthy adult liver, hepatocytes present a very low turnover. When a loss of hepatic mass occurs, as classically modeled by partial hepatectomy $(\mathrm{PH})$, it is rapidly compensated for by proliferation of mature hepatocytes. ${ }^{1}$ Several lines of experimental evidence support that mature hepatocytes are the main actors in this process and participation from intra- or extra-hepatic progenitor cells is negligible. ${ }^{1-5}$ Indeed, after the removal of $70 \%$ of rat liver mass $(70 \% \mathrm{PH})$, hepatocytes start to proliferate within $15 \mathrm{~h}$, peaking around $40-48 \mathrm{~h}$, and proliferation is maintained for 4 days. At this time, the remnant liver has regained $70 \%$ of the original liver weight. ${ }^{6}$ Further restoration of the liver mass is insured by hepatocytes hypertrophy, and complete restitution of liver mass occurs within 10 days post-hepatectomy.

Liver progenitor cells (LPC) are believed to represent a facultative reservoir for liver epithelial cells. In the healthy adult liver, LPC are a small population of undifferentiated cells, nested around the canal of Hering, the anatomical and physiological link between hepatocytes and terminal branches of the bile ducts. ${ }^{7}$ When activated, they proliferate and retain the capacity of differentiating into hepatocytes or into biliary cells. ${ }^{8-11}$ Such activation occurs when, in response to injury, the normal regenerative response through mature hepatocyte is either overwhelmed by the severity of injury, or

\footnotetext{
${ }^{1}$ Laboratory of Hepato-Gastroenterology, Institut de Recherche Expérimentale et Clinique, Université catholique de Louvain, Brussels, Belgium and ${ }^{2}$ Laboratory of Pediatric Hepatology \& Cell Therapy, Institut de Recherche Expérimentale et Clinique, Université catholique de Louvain, Brussels, Belgium

Correspondence: Professor IA Leclercq, MD, PhD, Laboratoire d'Hépato-Gastro-Entérologie, Institut de Recherche Expérimentale et Clinique, Université catholique de Louvain, GAEN53/79, Avenue Mounier, 53, B-1200 Brussels, Belgium.

E-mail: isabelle.leclercq@uclouvain.be

Received 28 March 2011; revised 11 July 2011; accepted 21 July 2011
} 
deficient due to replicative inability of hepatocytes to undergo cell division (a process known as replicative senescence). It has been reported that a threshold of a 50\% loss of hepatocytes, together with a significant decrease in proliferation of the remaining mature hepatocytes is required for LPC activation. ${ }^{12}$ Liver progenitor cell activation is encountered in most sub-acute and chronic liver diseases, and the extent of LPC proliferation has been shown to correlate with disease severity. ${ }^{13-15}$

LPC are a heterogeneous cell population identified as small oval cells expressing some biliary such as cytokeratins 7 and 19 (CK7 and CK19), or oval cell antigen 6, immature fetal hepatoblast (glutathione S-transferase protein (GSTp) and $\alpha$-fetoprotein) or stem cell markers (c-kit, stem cell antigen-1, CD133). ${ }^{16}$ Similar to bile duct cells, they also express the epithelial cell adhesion molecule (EpCAM), a factor that has been used to isolate adult rat LPC capable of repopulating an injured liver. ${ }^{17}$ While differentiating towards hepatocytes or cholangiocytes, LPC acquire the appearance of cells with intermediate phenotype. Such a transition is associated with a progressive loss of LPC markers while acquiring a more mature phenotype. ${ }^{18}$

The effective contribution of LPC to liver cell replacement is currently unknown. Seemingly, it requires two important processes, proliferation and progressive terminal differentiation. Undoubtedly, compared with classical regeneration involving mature hepatocytes, regeneration through LPC has been reported to be poorly efficient and lengthy. ${ }^{13,19,20}$ However, the very demonstration that LPC are capable of terminal differentiation into hepatocytes is leading to the investigation of the progenitor cell compartment of adult livers as a putative therapeutic target for liver regeneration. ${ }^{11}$

The aim of this work was to evaluate the contribution of LPCs to the recovery of liver mass and function in the 2-acetaminofluorene (AAF)/PH rat model.

\section{MATERIALS AND METHODS Animal Treatments}

The animal procedures were conducted according to the guidelines for humane care for laboratory animals established by the Universite catholique de Louvain (UCL), in accordance with European Union Regulation and protocols approved by the local ethic committee. Male Fisher 344 rats (7 weeks old) were purchased from Harlan (Horst, The Netherlands). After 1 week of acclimatization, 31 rats were implanted a AAF pellet $(25 \mathrm{mg}, 14$ days release, Innovative Research of America, Sarasota, FL, USA) subcutaneously. One week later, a sham operation (AAF/sham, $n=3)$ or a $70 \% \mathrm{PH}$ were performed (AAF/PH, $n=28$ ), as previously described. ${ }^{21}$ AAF/sham rats were killed 14 days post-sham and $\mathrm{AAF} / \mathrm{PH}$ rats were randomly assigned for killing at day 7 $(n=8)$, day $10(n=3)$ and day $14(n=17)$ post-PH. Untreated rats subjected to $\mathrm{PH}$ only ( $\mathrm{PH}, n=4)$ or not (CTL, $n=3$ ) were used as controls. In a second experiment, rats were subjected to $\mathrm{PH} 7,14$ or 21 days after the implantation of the AAF pellet ( $n=3,4$ and 4 , respectively). Liver regeneration $48 \mathrm{~h}$ after $\mathrm{PH}$ was evaluated and compared with that of PH rats at the same timing. In those, BrdU ( $1 \mathrm{mg}$ per $100 \mathrm{mg}$ body weight, ip) was injected $2 \mathrm{~h}$ prior to killing. The weight of the resected liver ( $\sim 70 \%$ of liver mass) was recorded. Blood was drawn by cardiac puncture at the time of killing and the liver rapidly excised and weighted. Small sections from inferior and superior right lobes were immediately snap-frozen in liquid nitrogen and kept at $-80^{\circ} \mathrm{C}$ until use. The remaining tissue was fixed in $4 \%$ formalin.

\section{Liver Mass and Function Recovery}

The rate of liver mass recovery (LMR) was estimated based on the following formula: $\%=100 \times \mathrm{Mf} / \mathrm{Mi}$; where $\mathrm{Mf}$ is the weight of liver at killing, and $\mathrm{Mi}$ is the total liver mass before hepatectomy estimated by dividing the mass of resected segments by 0.7 . The liver mass to body weight ratio was calculated at killing 14 days post $\mathrm{PH}$ in $\mathrm{AAF} / \mathrm{PH}(n=15)$ and in $\mathrm{PH}(n=4)$, and in AAF/sham $(n=3)$ and CTL $(n=3)$. Serum albumin and bilirubin concentrations were measured using automated procedures (Department of Clinical Biology, St Luc University Hospital, UCL, Brussels, Belgium) 14 days post $\mathrm{PH}$ in $\mathrm{AAF} / \mathrm{PH}(n=12)$ and in $\mathrm{PH}(n=4)$, as well as in $\mathrm{AAF} / \mathrm{sham}(n=3)$ and CTL $(n=3)$.

\section{Immunohistochemistry}

Paraffin-embedded livers were cut into $5-\mu \mathrm{m}$ thick sections. Hematoxylin and eosin (H\&E) staining was used for standard histology. For immunohistochemistry, mouse monoclonal antibodies against CK19 (Novocastra, Newcastle, UK; dilution 1:50), Ki67 (Dako, Glostrup, Denmark; dilution 1:50), 5-bromodeoxyuridine (Dako; dilution 1:100) and rabbit polyclonal antibody against GSTp (MBL International, Nakaku Nagoya, Japan; ready to use) were used. Detection was performed using the anti-mouse or anti-rabbit Envision system (Dako) as appropriate. Peroxidase activity was revealed by diamino-3, 3'-benzidine Substrate-Chromogen System (Dako) for CK19, Ki67 and BrdU, or by 3-amino9-ethylcarbazole Substrate-Chromogen System (Dako) for GSTp immunostaining. Slides were counterstained with hematoxylin.

\section{Morphometrical Analysis}

Images of sections stained for CK19 and Ki67 were taken at random at $\times 200$ magnification. Computer image analyses of immunostained sections were performed using Zeiss microscope coupled to an Axiocam camera (MR3, Carl Zeiss, Munich, Germany) and the Axiovision software (Zeiss), and morphometrical quantification was performed as previously described. $^{22}$ The percentage of $\mathrm{CK} 19^{+}$area was expressed as a ratio of stained area to the total image area (leaving out the white vascular areas). At least 10 images per section were analyzed. Consecutive sections stained with CK19 and Ki67 were superimposed to delineate the $\mathrm{CK} 19^{+}$area on 
Ki67-stained sections. $\mathrm{Ki}^{+} 7^{+}$nuclei were counted in $\mathrm{CK} 19^{+}$ and $\mathrm{CK} 19^{-}$area, on 10 images per liver slide. The number of stained nuclei to the total number of nuclei, in each CK19defined zone, was expressed as a percentage and the mean \pm s.d. percentage was calculated.

The number of CK19-positive intermediate hepatocytes or hepatocytes-like cells (with hepatocyte polygonal shape and low nuclear to cytoplasm ratio) was determined on at least five images per section and is expressed per surface unit.

Mitotic figures in hepatocytes were counted on minimum 15 high power fields per H\&E-stained liver section, and averaged. The BrdU hepatocyte labeling index and the Ki67 hepatocyte labeling index were estimated by counting hepatocyte positive nuclei on nine high-power field per section (a mean of 1500 hepatocytes per liver slide were counted), and were expressed as a percentage of hepatocyte-positive nuclei to the total number of hepatocytes.

\section{Real-Time Quantitative PCR}

Total RNA was extracted using TRIpure Isolation Reagent (Roche Diagnostics, Vilvoorde, Belgium). Reverse transcription and quantitative real-time PCR analysis were performed as previously described. ${ }^{22}$ Primer pairs for transcripts of interest were designed using the Primer Express design software (Applied Biosystems, Lennik, Belgium) and are listed in Table 1. RPL19 mRNA was chosen as an invariant standard. Results are expressed as fold expression relative to expression in control group (value set at 1) using the delta Ct method.

\section{Statistical Analysis}

Data are expressed as a mean \pm s.d. Statistical significance was determined by the Student $t$-test. $P$-values $<0.05$ were considered as statistically significant. For correlation, the Pearson test was used.

\section{RESULTS \\ Recovery of Liver Mass Is Impaired in AAF/PH Rats Despite LPC Expansion}

There was no mortality in the PH group or during the first 7 days post- $\mathrm{PH}$ in the AAF/PH groups. Two rats died at days 11 and 13 post- $\mathrm{PH}$, respectively. LMR at day 14 post $70 \% \mathrm{PH}$ was $\sim 100 \%$ in $\mathrm{PH}$ group. By contrast, in AAF/PH animals, LMR only reached $56.7 \pm 9.6,56.2 \pm 19.7$ and $67.2 \pm 15.8 \%$ at day 7 , day 10 and day 14 post- $\mathrm{PH}$, respectively, with no significant difference between time points (Figure 1a). The liver to body weight ratio at day 14 post- $\mathrm{PH}$ was lower in $\mathrm{AAF} / \mathrm{PH}$ than in $\mathrm{PH}$ only rats $(P=0.01)$, confirming impaired liver regeneration in this model (Figure 1b).

In CTL and AAF/sham livers, beside bile ducts, rare CK19positive cells were found directly adjacent to the portal tract. Those were more numerous in PH livers, without however reaching statistical significance (Figure 2a). In all $\mathrm{AAF} / \mathrm{PH}$ livers, the number of $\mathrm{CK} 19^{+}$cells was significantly increased. Those formed pseudoductular structures expanding from the portal tract towards the centrolobular zone (Figure 2a). As assessed by morphometrical analysis of CK19-immunostained sections, there was a stepwise increase in the magnitude of $\mathrm{CK}_{1} 9^{+}$cell expansion between day 7 and day 14 post-hepatectomy, in the AAF/PH group with a large interindividual variability at each time point (Figure 2b). Similarly, CK19 mRNA expression was increased in AAF/PH livers compared with CTL, AAF/sham or with $\mathrm{PH}$ only, with no statistical difference between day 7 and day 14 (Figure 2c).

\section{In AAF/PH Liver, Liver Progenitor Cell Expansion Is Inversely Correlated to the Recovery of Liver Mass and Function}

In CTL animals, serum albumin concentration was at $1.31 \pm 0.13 \mathrm{~g} / \mathrm{dl}$ and serum bilirubin concentration at $0.49 \pm 0.22 \mathrm{mg} / \mathrm{dl}$. PH or AAF treatment as in AAF/sham did not alter albumin or bilirubin levels. Out of $12 \mathrm{AAF} / \mathrm{PH}$ rats in which blood was available for analysis, $9 \mathrm{AAF} / \mathrm{PH}$ rats had levels of bilirubin 14 days post- $\mathrm{PH}(0.41 \pm 0.08)$ in a range

Table 1 List of primer sequences used in the study

\begin{tabular}{|c|c|c|}
\hline \multirow[t]{2}{*}{ Genes } & \multicolumn{2}{|c|}{ Primer sequence } \\
\hline & Forward & Reverse \\
\hline Albumin & 5'-GCCTGGGCAGTAGCTCGTAT-3' & 5'-CTGTTGCCAATTGGTGATTC-3' \\
\hline CD133 & 5'-CTTGGCATCGCGTTGG-3' & 5'-TGCCAGCTTCTGGGTCCTT-3' \\
\hline CK19 & 5'-GAACTCCAAGATAGTCCTACAGATCGA-3' & 5'-GTCTCAAACTTGGTCCGGAAGT-3' \\
\hline IGF-I & 5'-AACAAGCCCACAGGCTATGG-3' & 5'-AAGCAACACTCATCCACAATGC-3' \\
\hline OTC & 5'-CAATATCCTGCACTCCATCATGAT-3' & 5'-GGCTCATAACCCTITGGAGTAGCT-3' \\
\hline RPL 19 & 5'-CAAGCGGATTCTCATGGAGCA-3' & 5'-TGGTCAGCCAGTAGCTTCTT-3' \\
\hline TGF $\beta 1$ & 5'-AGAAGTCACCCGCGTGCTA-3' & 5'-TGTGTGATGTCTTGGTITGTCA-3' \\
\hline
\end{tabular}


a

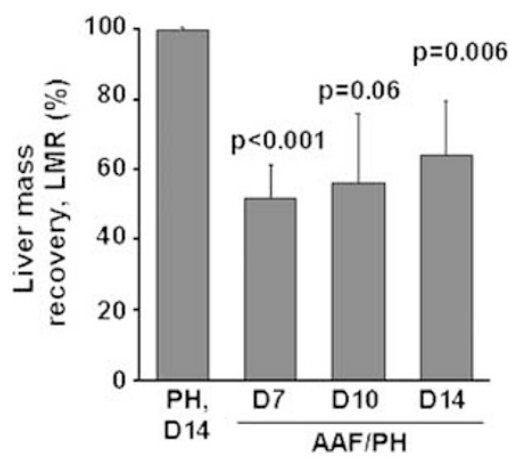

b

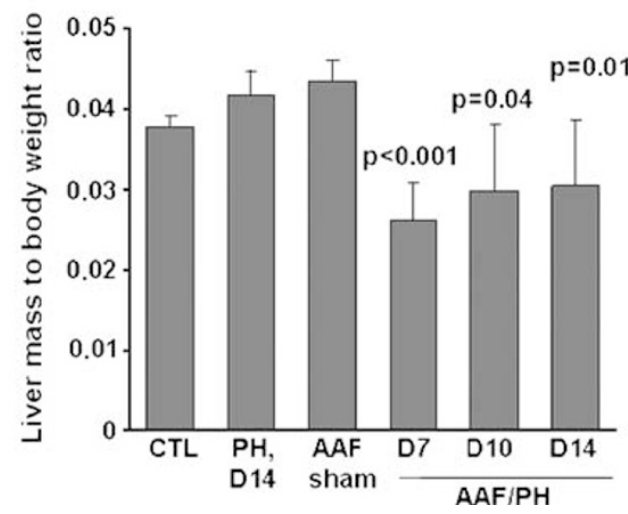

Figure 1 Evaluation of the liver regeneration after partial hepatectomy (PH). (a) Graph representing the estimated liver mass recovery (LMR) post-PH in rats subjected to $\mathrm{PH}$ alone 14 days after $\mathrm{PH}$, and in 2-acetaminofluorene (AAF)/PH rats $7(n=8), 10(n=3)$ and 14 days post-PH (n=15). (b) Graph representing the liver mass to body weight ratio calculated for the same groups and for AAF/sham rats. $P$-values are for comparison with $\mathrm{PH}$ group 14 days post-PH. $P$-values $<0.05$ were considered as statistically significant.

similar to that of controls with slightly decreased albumin levels $(1.2 \pm 0.08, P=0.0006)$. By contrast, in the remaining three, albumin levels fell below the detection limit $(<0.7 \mathrm{~g} /$ $\mathrm{dl}$ ) and bilirubin levels were dramatically increased (above $1.3 \mathrm{mg} / \mathrm{dl}$; Figures $3 \mathrm{a}$ and $\mathrm{b}$ ). Thus, despite impaired LMR, liver function was grossly preserved in 9/12 rats, but defective in $3 / 12$ rats.

We then correlated serum albumin and bilirubin levels with LMR and observed that failure of maintaining liver function was associated with a lower LMR $(45.1 \pm 4.9 \%)$, compared with $\mathrm{AAF} / \mathrm{PH}$ rats with albumin and bilirubin in the near normal range, where LMR was significantly higher $(74.6 \pm 9.4 \%, \quad P=0.0005$; Figures $3 \mathrm{a}$ and $\mathrm{b})$. Although similar in $\mathrm{PH}, \mathrm{AAF} / \mathrm{sham}$ and $\mathrm{AAF} / \mathrm{PH}$ rats with normal liver function, the liver mass to body weight ratio was significantly lower in AAF/PH rats with deficient liver function (Figure 3c). Consistent with low serum levels, hepatic albumin mRNA expression was drastically reduced in AAF/PH rats with low LMR (Figure 3d). Of note, as in AAF/sham livers, hepatic albumin mRNA was significantly downregulated to $\sim 40 \%$ of control values in $\mathrm{AAF} / \mathrm{PH}$ rats with higher LMR, although serum albumin was only moderately reduced. IGF-1 mRNA followed a similar pattern of expression (not shown). Also, liver OTC mRNA expression was significantly reduced in $\mathrm{AAF} / \mathrm{PH}$ rats with $\mathrm{LMR}<56 \%$ compared with those with a better LMR or with AAF/Sham (Figure 3e).

Next we evaluated LPC expansion in relation to LMR and liver function 14 days post-PH. As already shown, expansion of $\mathrm{CK} 19^{+}$cells was minimal in $\mathrm{PH}$ livers (Figure 2b). For $\mathrm{AAF} / \mathrm{PH}$ rats, we found that LPC expansion, as assessed by CK19 morphometry, was inversely correlated with LMR $\left(R^{2}=0.63, P<0.0001\right.$; Figure $\left.4 \mathrm{a}\right)$. Thus, in livers that poorly recovered their mass and had a deficient function, LPC were significantly more numerous $(17.4 \pm 4.1$ vs $3.1 \pm 1.5$ of CK19 positive area, $P<0.0001$ ), spreading from the periportal and the entire mid-zonal area in livers. In those with a better
LMR (above 56\%) and liver function, progenitor cell expansion was restricted to the periportal zone. Hepatic CK19 mRNA expression varied in a similar fashion (Figure 4b), being significantly more overexpressed in AAF/PH liver with poor function and LMR than in those with preserved function and better LMR.

Transcript levels of CD133, described as a marker of nondifferentiated progenitor cells, ${ }^{16,23}$ and transforming growth factor- $\beta 1$ (TGF- $\beta 1$ ), a known mitogen for LPC, ${ }^{24}$ were significantly higher in AAF/sham and AAF/PH livers with poor LMR, compared with those with higher LMR (Figures 4c and d). We additionally observed an overexpression of CD133 mRNA in PH rats, compared with the untreated CTL livers.

As LPC differentiation into functional hepatocyte is believed to occur as a rescue mechanism to compensate for defective liver function, we searched for signs of hepatocytic differentiation of LPC in AAF/PH livers. $\mathrm{CK}_{19}{ }^{+}$or GSTp $^{+}$/ $\mathrm{CK} 19^{-}$morphologically resembling hepatocytes (hepatocytelike cells or intermediate hepatocytes; Figure 5a) were quasi absent in $\mathrm{AAF} / \mathrm{PH}$ livers with $\mathrm{LMR}>56 \%$ and low expansion of LPC (Figure 5b). Although slightly more numerous, they remain scarce (less than 1 cell per $\mathrm{cm}^{2}$ ) in livers with low LMR and defective function (Figure 5b). Transcripts levels of hepatocyte growth factor, a growth factor involved in LPC differentiation, ${ }^{25}$ were found to be slightly, but not significantly, higher in AAF/PH livers with $\mathrm{LMR}<56 \%$ (Figure 5c).

\section{In AAF/PH Livers, Maintenance of Liver Function Depends Rather on Hepatocyte Replication than on LPC Hepatic Differentiation}

CK19 and Ki67 immunostainings were performed on serial sections to identify cycling $\mathrm{Ki}^{+} 7^{+}$cells and results confirmed by double immunofluorescence (not shown). At day 14 posthepatectomy, in $\mathrm{PH}$ group, $\mathrm{Ki}^{6} 7^{+}$nuclei were very few and belonged quasi exclusively to hepatocytes (Figures $6 a$ and $b$ ). 

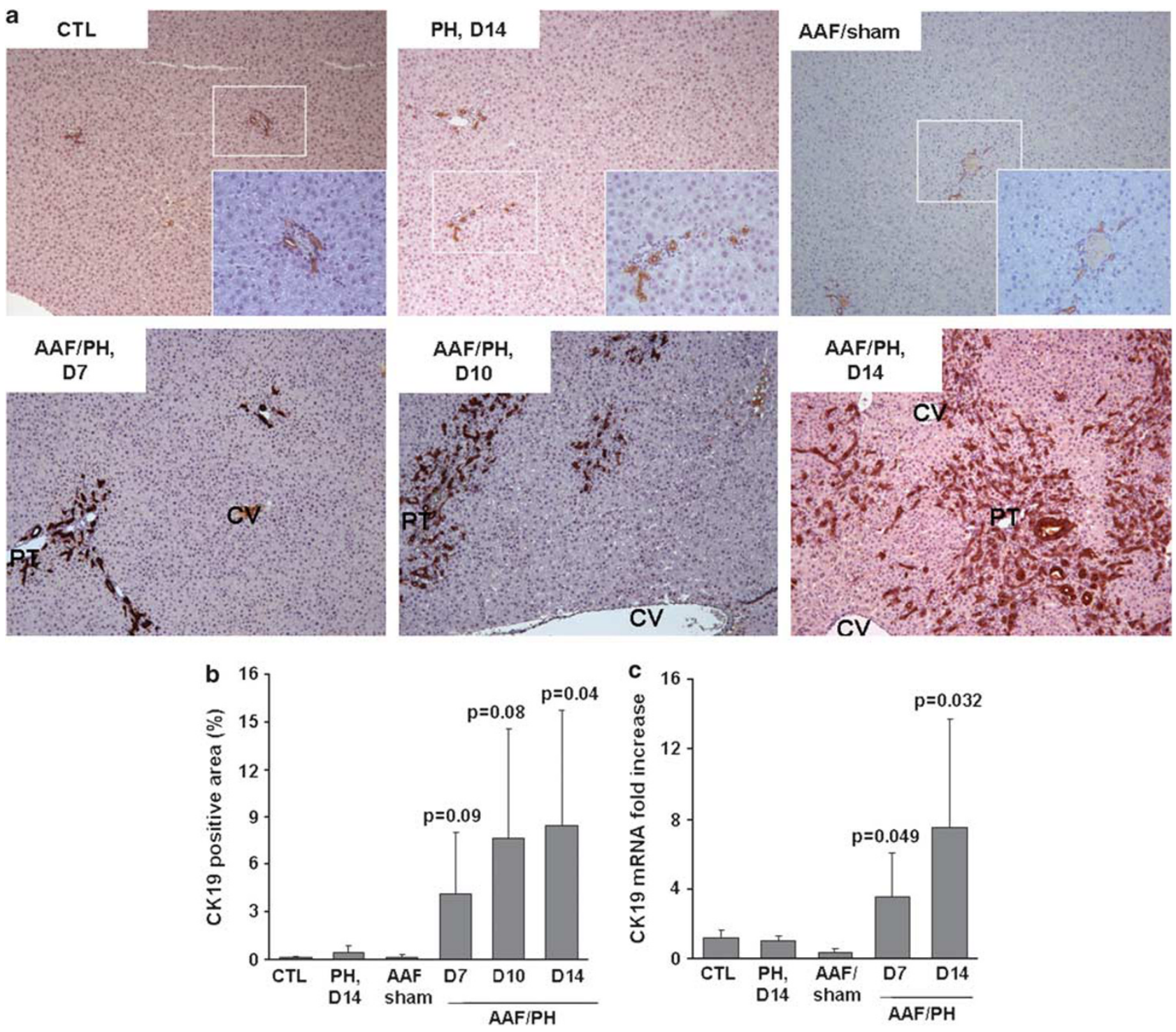

Figure 2 Liver progenitor cells (LPC) expansion. (a) Representative pictures of cytokeratin 19 (CK19) immunostaining: in untreated (CTL) liver, 2-acetaminofluorene (AAF)/sham liver, partial hepatectomy (PH) liver 14 days post-PH, and in AAF/PH livers 7, 10 and 14 days post-PH. Magnification $\times 100$ (PT = portal tract, CV = central vein). (b) Graph representing morphometrical analysis of CK19-stained sections of CTL livers ( $n=3)$, AAF/sham livers $(n=3), \mathrm{PH}$ livers 14 days post-PH $(n=4)$, and AAF/PH livers $7(n=8), 10(n=3)$ and $14(n=15)$ days post-PH. Note the large interindividual variability in $\mathrm{CK}_{19}{ }^{+}$cell expansion at each time point. (c) Graph showing CK19 mRNA levels analyzed in the same groups. $P$-values are for comparison with PH group 14 days post-PH. $P$-values $<0.05$ were considered as statistically significant.

In $\mathrm{AAF} /$ sham as in CTL livers, $\mathrm{Ki}^{+}{ }^{+}$hepatocyte nuclei were quasi absent. The proportion of $\mathrm{Ki}^{+} 7^{+}$nuclei was significantly higher in AAF/PH livers, and even more in those with poor function and LMR (Figure 6b). In AAF/PH livers with poor LMR, cycling cells were in majority small oval cells in CK19-positive areas (Figures 6a and b). In AAF/PH with preserved liver function and better mass recovery, besides small Ki67 ${ }^{+}$cells being found in CK19-positive area, numerous pericentral $\mathrm{Ki} 67^{+}$hepatocytes were also observed in pericentral areas (Figures $6 a$ and $b$ ). It thus appears that, in $\mathrm{AAF} / \mathrm{PH}$ livers, the limitation of the expansion of LPC, as well as the maintenance of liver function, are both related to the proliferative capacity of hepatocytes.
To evaluate the possibility that hepatocytic proliferation may result from a fading or a termination of AAF effect, rats were implanted an AAF pellet and subjected to $\mathrm{PH} 7,14$ or 21 days later. The proliferative response was analyzed $48 \mathrm{~h}$ post-PH and compared with that of control rats. As shown in the Supplementary Figures, liver regeneration was deeply inhibited in rats exposed to AAF, even when $\mathrm{PH}$ was performed 21 days after pellet implantation. Indeed, contrasting with prominent hepatocytic proliferation in $\mathrm{CTL} / \mathrm{PH}$ rats (group A), mitotic figures were absent in hepatocytes, and BrdU and Ki67 hepatocyte labeling indices were low and not significantly different from untreated livers. Of note, at this early time point after $\mathrm{PH}, \mathrm{CK} 19^{+}$LPC proliferate actively in 

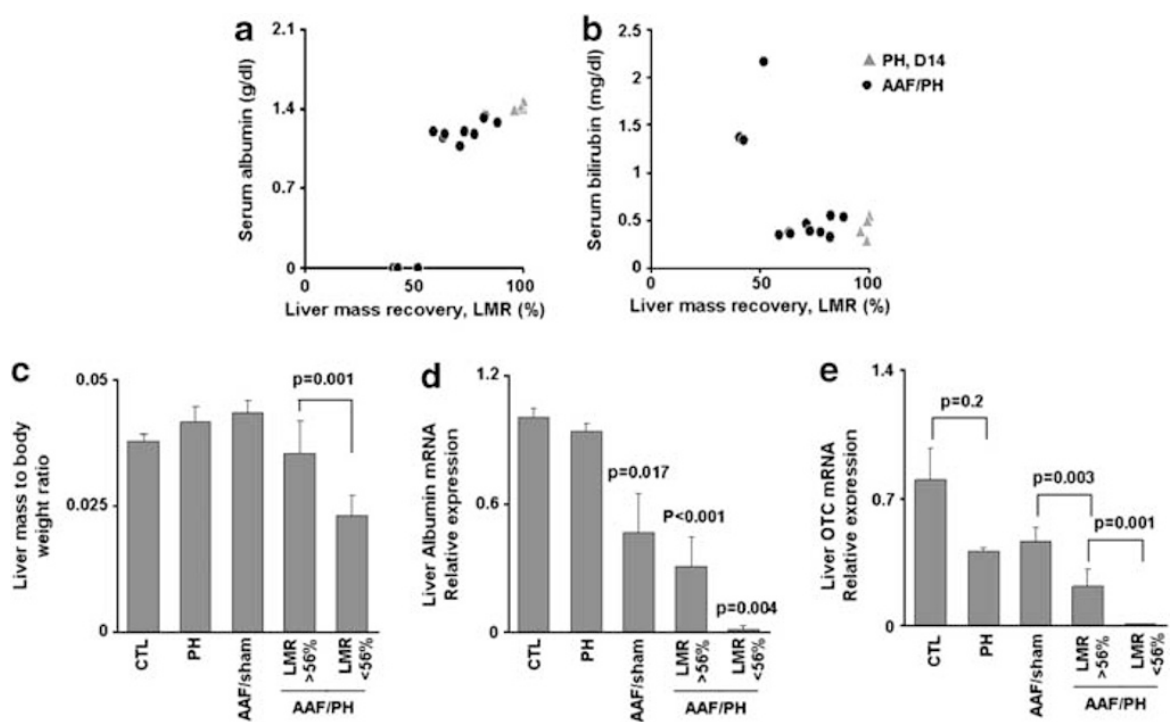

Figure 3 Correlation between liver mass recovery (LMR) and liver function. Graphs representing serum albumin (a) and serum bilirubin (b) levels measured in partial hepatectomy $(\mathrm{PH})$ and 2-acetaminofluorene (AAF)/PH rats at day 14 post-hepatectomy, and correlated to the LMR. Note that serum albumin levels below the detection limit of $0.7 \mathrm{~g} / \mathrm{dl}$ are arbitrary depicted as 0 . (c) Graph representing liver mass to body weight ratio calculated at sacrifice for CTL rats, PH rats, AAF/sham rats, and for AAF/PH rats with $\mathrm{LMR}>56 \%(n=9)$ or $\mathrm{LMR}<56 \%(n=6) 14$ days after surgery (PH or sham). (d) Graph showing the hepatic mRNA expression of albumin ( $P$-values are for comparison with PH group 14 days post-PH) and (e) OTC analyzed by RT-qPCR for the same groups. $P$-values $<0.05$ were considered as statistically significant.

all three $\mathrm{AAF} / \mathrm{PH}$ time-point groups confirming that delivery of AAF though the pellets inhibits, efficiently and for a prolonged period, liver regeneration, enabling proliferation of progenitors.

\section{DISCUSSION}

When hepatocyte replication is impaired, liver regeneration is believed to occur through proliferation and progressive terminal differentiation of liver progenitor cells. ${ }^{26}$ Here we used rats exposed to AAF and submitted to a $\mathrm{PH}$ as a model in which hepatocyte proliferation is impaired and LPC response stimulated. ${ }^{14,16}$ As expected, in those conditions, liver regeneration is poor and LPC expand. Twenty five \% of rats presented impaired liver function and defective LMR. In such livers, we found a high proliferation of LPC, but not of hepatocytes, and poor signs of LPC differentiation towards hepatocytes. By contrast, in $75 \%$ of the rats, liver function was relatively preserved and partial LMR was associated with poor LPC expansion and with hepatocyte proliferation. Thus, a high expansion of LPC correlated with a poor recovery of liver function and the contribution of hepatocytic differentiation of LPC to hepatic function was negligible. Our data support that, in this model of impaired liver regeneration, proliferation of the remnant hepatocytes rather than differentiation of LPC contributes to maintenance of liver function.

AAF is metabolized by cytochrome $\mathrm{P} 450$ into hydroxylated metabolites that bind to DNA, hereby blocking cell cycle progression before $\mathrm{S}$ phase and impeding cell function, in hepatocytes but not in LPC that lack such enzymes. ${ }^{26}$ As abundantly documented, hepatocyte proliferation peaks $48 \mathrm{~h}$ after $\mathrm{PH}$, leading to complete regeneration of the liver mass within 10 days after $70 \% \mathrm{PH}$ in healthy adult rats, through a mechanism that engages proliferation of mature remnant hepatocytes. ${ }^{1,6,11}$ In AAF/PH livers, the replicative ability of hepatocytes being impaired, the physiological regenerative response to $\mathrm{PH}$ does not occur. This is confirmed in our system as hepatocyte proliferation was completely abrogated $48 \mathrm{~h}$ post- $\mathrm{PH}$ in AAF-treated rats, irrespectively of the duration of exposure to AAF before $\mathrm{PH}$. By day 14 post-PH, a bare $30 \%$ recovery of the residual mass was reached in rats exposed to AAF compared with $70 \%$ in control $\mathrm{PH}$ rats, and there was no significant increment in LMR between day 7 and 14 post-PH. In AAF/PH livers, proliferative $\mathrm{CK} 19^{+}$LPC were already seen $48 \mathrm{~h}$ post $\mathrm{PH}$, and with time, further expand to form pseudoductular structures extending from the portal tract. As those cells are able to engage into hepatocyte lineage differentiation, such a LPC response constitutes a significant potential reservoir for hepatocytes. Consistent with previous reports, ${ }^{27}$ in $\mathrm{AAF} / \mathrm{sham}$ livers, neither the ductular reaction (expansion of $\mathrm{CK} 19^{+} \mathrm{LPC}$ ) nor the hepatocyte proliferation response were initiated, assuming that LPC response requires both the loss of liver mass (here by $\mathrm{PH}$ ) and the impairment of hepatocyte replication.

Adding to the $12 \%$ mortality (2/17), $25 \%$ of surviving $\mathrm{AAF} / \mathrm{PH}$ rats had a liver failure 14 days post- $\mathrm{PH}$, as assessed by serum albumin and bilirubin levels, whereas the others maintained liver function. We first hypothesized that preserved function and regeneration might result from 
a

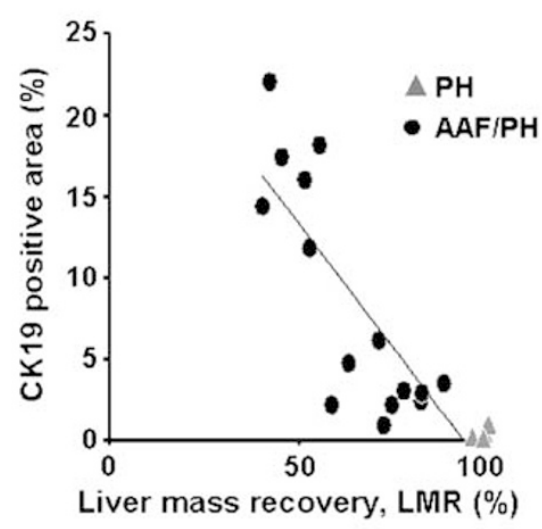

b
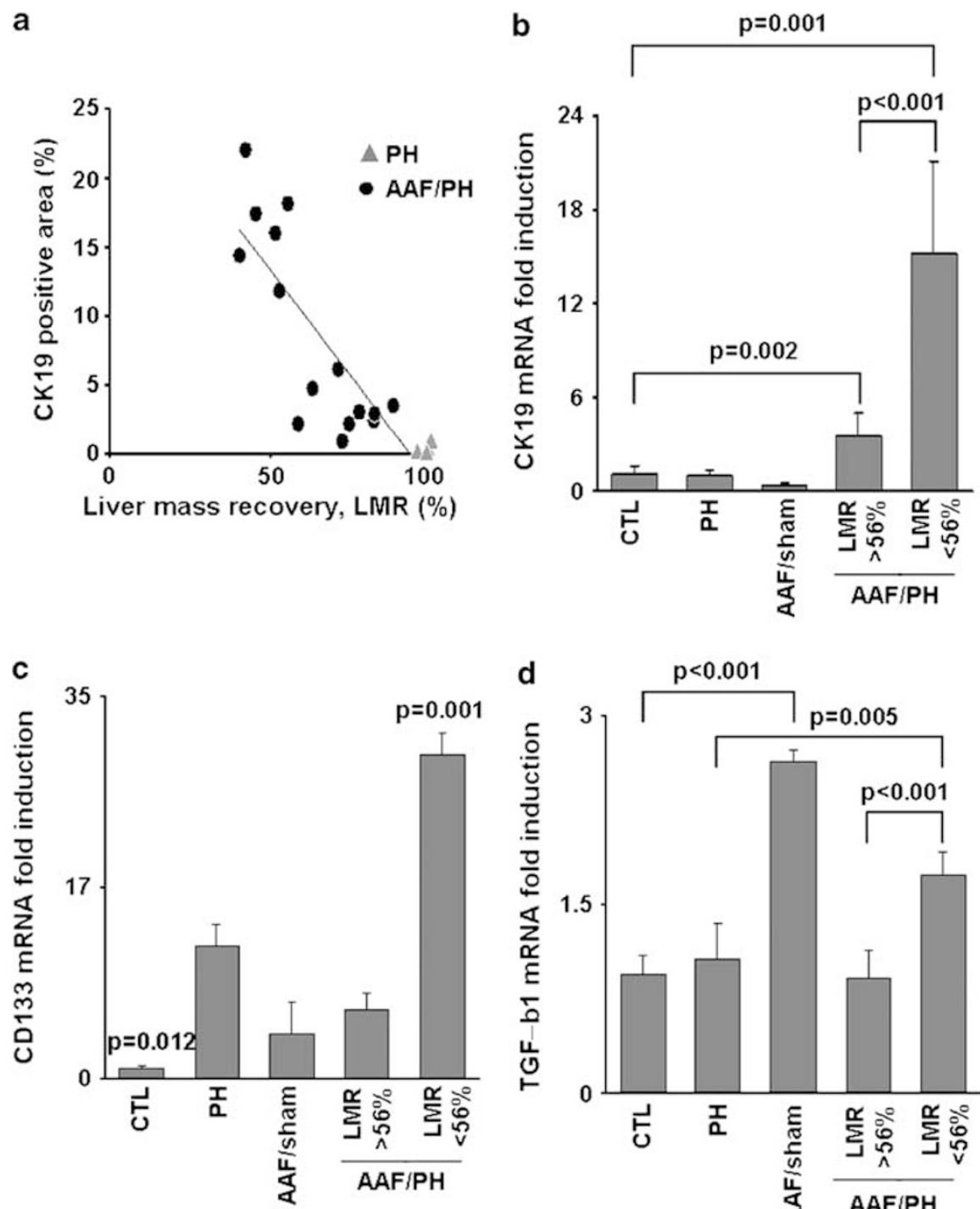

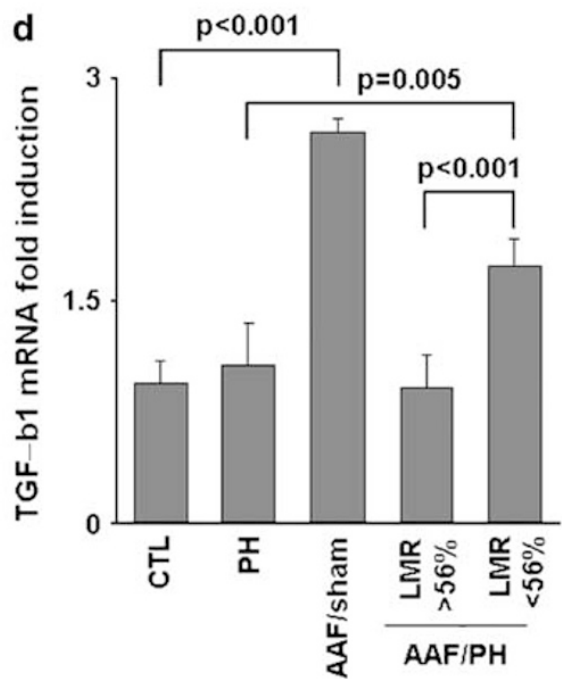

Figure 4 Inverse correlation between liver progenitor cells (LPC) expansion and liver mass recovery (LMR). (a) Graph representing cytokeratin 19 (CK19) ${ }^{+}$ area (assessed by morphometrical analysis of CK19 immunostaining) correlated to LMR in PH $(\mathbf{\Delta})$ and AAF/PH $(\mathbf{O})$ animals at day 14 post-PH. $\left(R^{2}=0.63\right.$, $P<0.0001)$. Graphs showing the hepatic transcript levels of (b) CK19, (c) CD133 ( $P$-values are for comparison with PH group 14 days post-PH), and (d) transforming growth factor- $\beta 1$ (TGF- $\beta 1$ ) in non-treated CTL livers, in $\mathrm{PH}, \mathrm{AAF} / \mathrm{sham}$ and AAF/PH (with $\mathrm{LMR}>56 \%$ or $\mathrm{LMR}<56 \%$ ) livers, at day 14 posthepatectomy. $P$-values $<0.05$ were considered as statistically significant.

a terminal differentiation of $\mathrm{CK} 19^{+}$progenitor cells into hepatocytes. We effectively observed an inverse correlation between expansion of $\mathrm{CK}_{1} 9^{+}$cells and both LMR and function. CK19 as well as other progenitor markers being progressively lost during differentiation, ${ }^{18,28,29}$ was thus also compatible with increased differentiation. However, in AAF/ PH livers, we identified very few hepatocyte-like cells. Those were characterized by maintenance of LPC markers, together with acquisition of hepatocyte-like morphology which represent an intermediate phenotypic state between $\mathrm{CK} 19^{+}$ small oval LPC and terminally differentiated hepatocytes. ${ }^{14,18,30}$ In addition, the number of intermediated cells was lower in livers with better function than in those with failure. Therefore, maintenance of liver function and LMR does not appear to rely on hepatocytic differentiation of LPC in this AAF/PH model. We next evaluated proliferating cells and found numerous $\mathrm{Ki} 67^{+}$hepatocyte nuclei and mitosis (not shown) in the pericentral areas (ie away from the periportal zone where the LPC arise from) in those livers with better function and lower LPC. By contrast in poorly regenerating livers, proliferating cells were quasi exclusively located in $\mathrm{CK}_{1} 9^{+}$LPC area and rarely corresponded to hepatocytes. This supports that, in our AAF/PH rats, liver mass gain ensued from hepatocyte proliferation and was sufficient for the maintenance of liver function. In the absence of such a process, hepatocyte mass did not increase sufficiently, and liver function failed despite an increased proliferation and attempt of differentiation of LPC. 
a

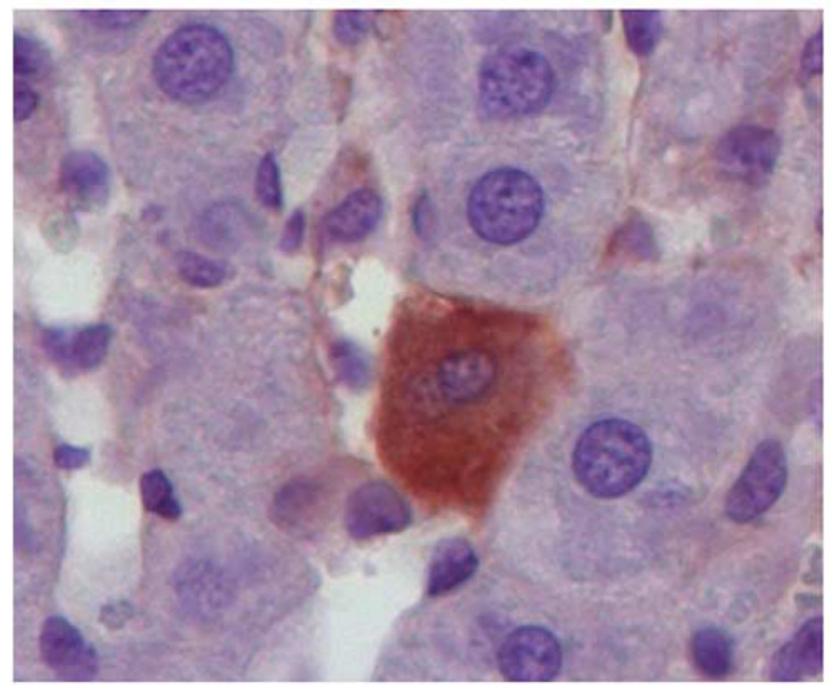

b

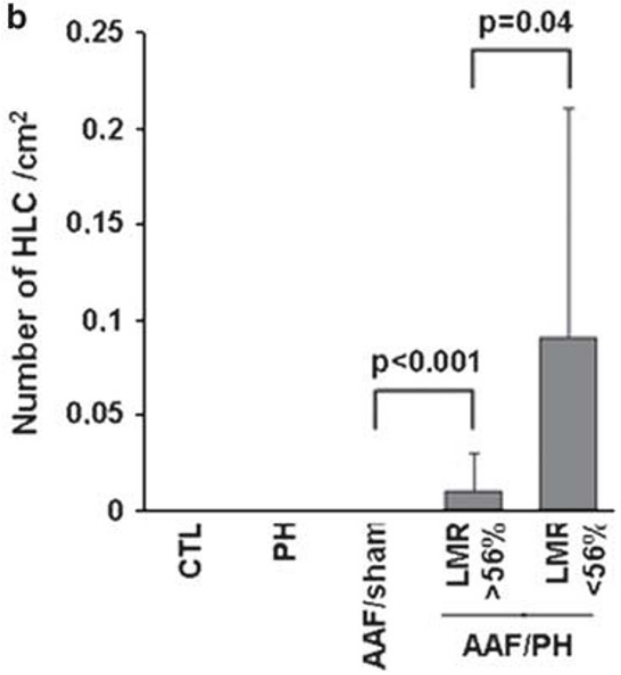

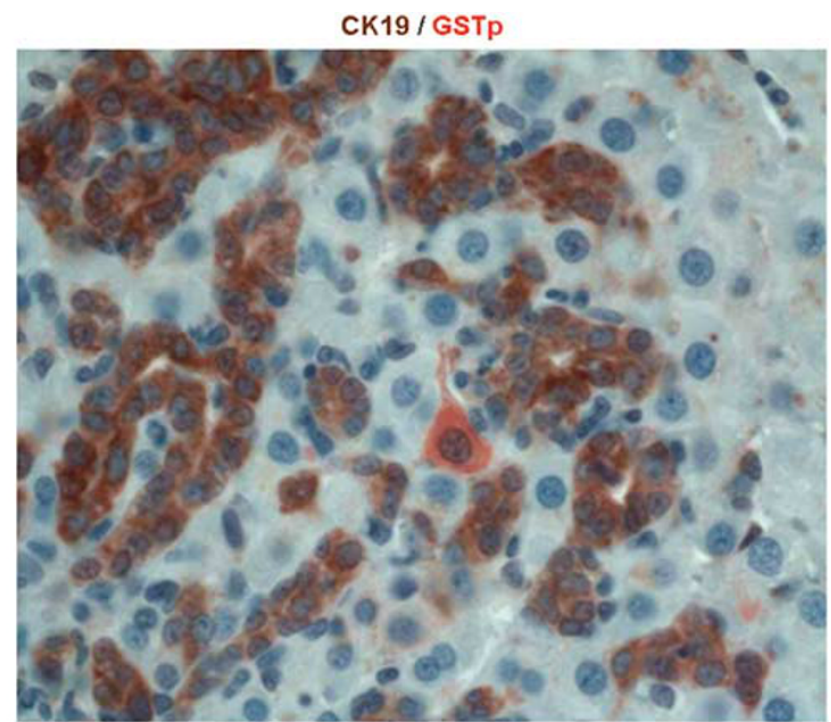

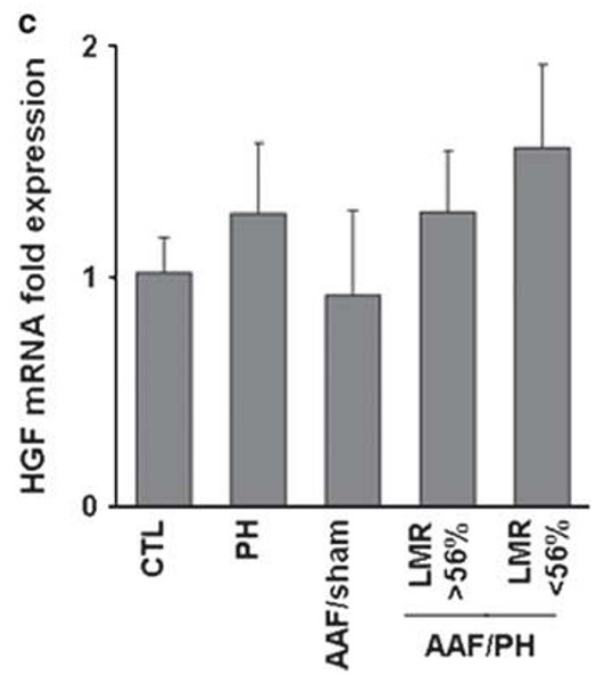

Figure 5 Rare signs of hepatic differentiation of liver progenitor cells (LPC). (a) Pictures of cytokeratin 19 (CK19) and CK19/glutathione S-transferase protein (GSTp) immunohistochemistry in 2-acetaminofluorene/partial hepatectomy (AAF/PH) liver with liver mass recovery (LMR) $<56 \%$ demonstrating CK19 ${ }^{+}$ hepatocytes-like cells (HLC, with hepatocyte polygonal morphology) (Magnification $\times 400)$ or GSTp $^{+} /$CK19 $^{-}$intermediate hepatocytes (CK19 in brown, GSTp in red. Magnification $\times 200$ ). (b) Graphs representing the number of CK19 ${ }^{+} \mathrm{HLC}$ per $\mathrm{cm}^{2}$ of liver section in CTL, AAF/sham, PH and AAF/PH livers with $\mathrm{LMR}>56 \%$ or $\mathrm{LMR}<56 \%, 14$ days post-PH. (c) Representation of hepatic hepatocyte growth factor (HGF) mRNA expression analyzed by RT-qPCR in the same groups. $P$-values $<0.05$ were considered as statistically significant.

The reasons why, although delayed, hepatocyte proliferation occurs in some AAF/PH livers are not clear. Reduced availability of AAF because of incomplete or delayed emptying of the pellet is plausible. However, all pellets had similar macroscopic and microscopic appearance and weight at the time of killing, and no local inflammation or collections at the site of implantation were observed. The expression of hepato-specific genes such as albumin, OTC or IGF-1 significantly decreased in all AAF livers, whether with sham or $\mathrm{PH}$, (although more dramatically in $\mathrm{AAF} / \mathrm{PH}$ with LMR $<56 \%$ ), suggesting AAF-induced hepatotoxicity in all rats exposed to AAF. Also, we did not observe a fading or a termination of the AAF effect in the time frame used in our experiments since peak of hepatocytic proliferation remained completely abrogated $48 \mathrm{~h}$ post-PH in rats even when $\mathrm{PH}$ was performed 21 days after the implantation of the AAF pellet. Despite the deep inhibition of hepatocytic proliferation in the acute phase after $\mathrm{PH}$, with time, hepatocytes do manage to escape this break, at least in some AAF-treated livers. Whether this reflects an interindividual susceptility to AAF, a variability in the process of escape from hepatocyte mitogenic blockade or in a compensatory, overstimulation of the environmental mitotic pressure remains to be established. The observation of decreased expression of TGF- $\beta 1$, a well known mito-inhibitor for hepatocytes, in those livers in which hepatocyte proliferation is eventually seen, and in 

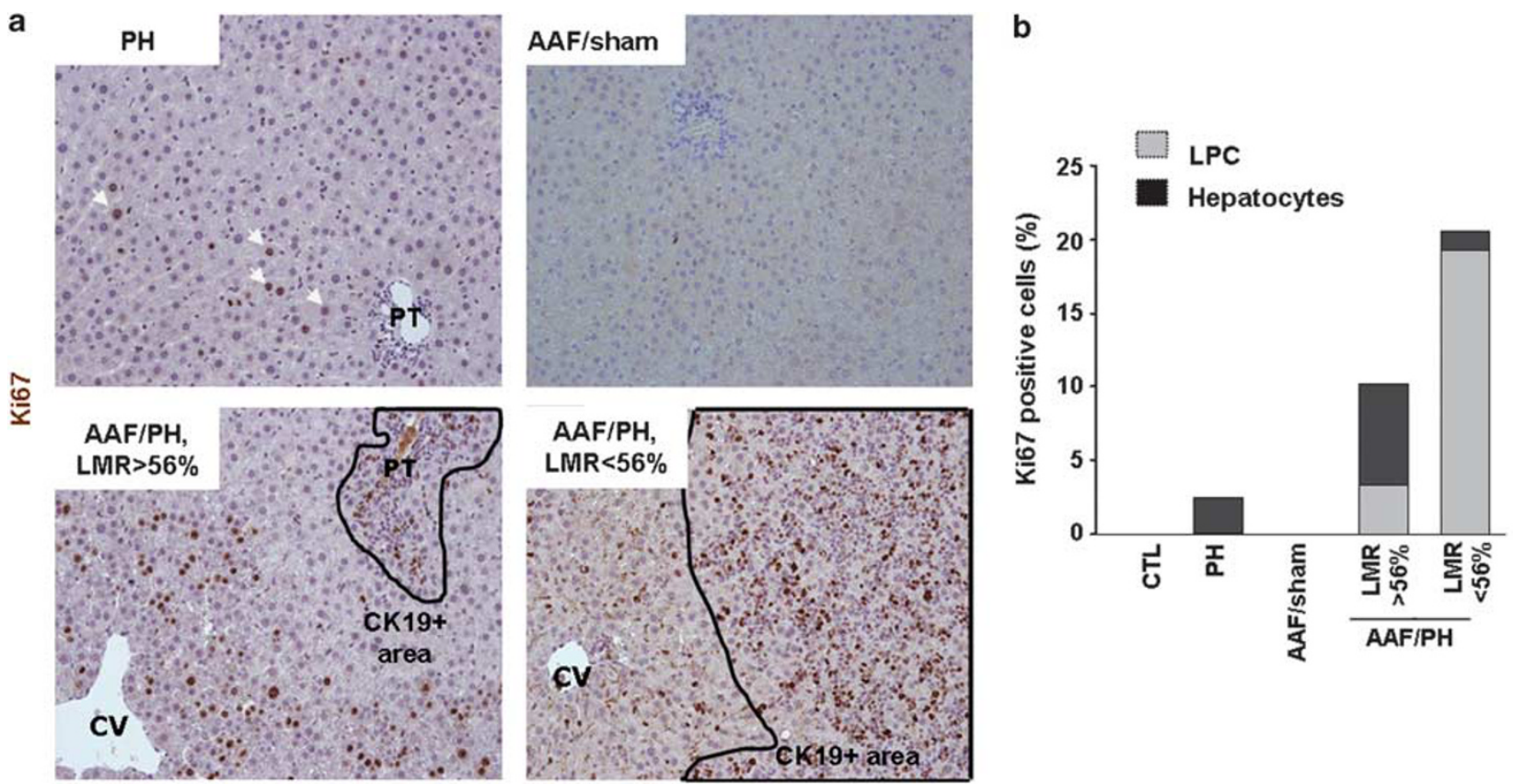

Figure 6 Hepatic cell proliferation in periportal and pericentral areas correlated to liver mass recovery (LMR). (a) Representative pictures of liver sections of partial hepatectomy (PH), 2-acetaminofluorene (AAF)/sham, and AAF/PH animals with LMR $>56 \%$ and LMR $<56 \%, 14$ days post-PH or sham, labeled with Ki67 antibody (brown nuclear staining). Cytokeratin 19 (CK19)-positive areas assessed on consecutive sections are delineated by the continuous lines $\left(\mathrm{CV}=\right.$ central vein, PT = portal tract). (b) Graph representing the Ki67 labeling index (as the percentage of Ki67 ${ }^{+}$cells) in CK19-positive liver progenitor cell (LPC) area (grey bars), and in CK19-negative area (black bars), in CTL and AAF/sham livers, and at day 14 post-PH in PH and AAF/PH with LMR $>56 \%$ or $\mathrm{LMR}<56 \%$.

which some recovery of liver mass occurs, provides support to the latter.

Several cytokines and growth factors have been implicated in the control of proliferation and differentiation of LPC. ${ }^{25,31,32}$ In AAF/PH livers, lack of hepatocyte proliferation and liver failure are associated with maximal proliferation of LPC, but poor differentiation. In PH alone, increased CD133 expression, a stem cell marker expressed by immature progenitors, has been shown to correspond to recruitment of $\mathrm{CD}_{133^{+}}$bone marrow-derived sinusoidal progenitors. ${ }^{33}$ In $\mathrm{AAF} / \mathrm{PH}$ livers with large expansion of LPC, increased expansion of CD133 is compatible with the increased presence of immature progenitors. Whether progenitors other than LPC are also recruited, remains to be determined. The pattern of LPC suggests that, the cocktail of signals in failing livers efficiently stimulates proliferation of LPC, but not their differentiation. Cytokines such as TGF- $\beta 1$ and oncostatin M (OSM) might be part of this cocktail. TGF- $\beta 1$ was upregulated in $\mathrm{AAF} / \mathrm{PH}$ livers, but not sufficiently to improve LPC differentiation, in our experimental conditions. Unlike hepatocytes, LPC are resistant to the mito-inhibitory effect of TGF- $\beta 1^{34,35}$ and in specific context, TGF- $\beta$ has been shown to favor cell differentiation. ${ }^{32,36}$ Similarly, Okaya et al ${ }^{37}$ demonstrated the induction of the OSM receptor (OSM-R) in $\mathrm{AAF} / \mathrm{PH}$ model while OSM induces LPC differentiation in vitro.

In conclusion, we demonstrated that, in this model, LPC proliferation is not associated with restoration of liver function and mass, because they poorly engage into differentiation. Whether stimulation of hepatocytic lineage differentiation would rescue function in failing livers remains to be ascertained, in which case, the challenge ahead is to identify means to favor functional differentiation as tools to salvage acute and chronic liver failure.

Supplementary Information accompanies the paper on the Laboratory Investigation website (http://www.laboratoryinvestigation.org)

\section{ACKNOWLEDGEMENTS}

We are thankful to Dr Stephane Eeckhoudt from the Department of Clinical Biology, St Luc University Hospital, UCL, Brussels, for measurement of serum albumin and bilirubin levels, and to Regina Español Suñer for the critical review of the manuscript. This research was supported by grants from the Belgian Federal Science Policy Office (Interuniversity Attraction Poles program - network P6/36) and the Brussels Capital Region (Impulse programme-Life Sciences 2007; BruStem project). IL is a FRS-FNRS research associate.

\section{DISCLOSURE/CONFLICT OF INTEREST}

The authors declare no conflict of interest.

1. Fausto N. Liver regeneration and repair: hepatocytes, progenitor cells, and stem cells. Hepatology 2004;39:1477-1487.

2. Fausto N, Campbell JS, Riehle KJ. Liver regeneration. Hepatology 2006:43:S45-S53.

3. Grisham JW. A morphologic study of deoxyribonucleic acid synthesis and cell proliferation in regenerating rat liver; autoradiography with thymidine-H3. Cancer Res 1962;22:842-849. 
4. Fujii $\mathrm{H}$, Hirose $\mathrm{T}$, Oe $\mathrm{S}$, et al. Contribution of bone marrow cells to liver regeneration after partial hepatectomy in mice. J Hepatol 2002;36:653-659.

5. Beaudry $P$, Hida $Y$, Udagawa $T$, et al. Endothelial progenitor cells contribute to accelerated liver regeneration. J Pediatr Surg 2007;42: 1190-1198.

6. Martins PN, Theruvath TP, Neuhaus P. Rodent models of partial hepatectomies. Liver Int 2008;28:3-11.

7. Kuwahara R, Kofman AV, Landis $C S$, et al. The hepatic stem cell niche: identification by label-retaining cell assay. Hepatology 2008;47:1994-2002.

8. Alison MR, Vig P, Russo F, et al. Hepatic stem cells: from inside and outside the liver? Cell Prolif 2004;37:1-21.

9. Evarts RP, Nagy $\mathrm{P}$, Nakatsukasa $\mathrm{H}$, et al. In vivo differentiation of rat liver oval cells into hepatocytes. Cancer Res 1989;49:1541-1547.

10. Golding $M$, Sarraf $C E$, Lalani EN, et al. Oval cell differentiation into hepatocytes in the acetylaminofluorene-treated regenerating rat liver. Hepatology 1995;22:1243-1253.

11. Alison MR, Islam S, Lim S. Stem cells in liver regeneration, fibrosis and cancer: the good, the bad and the ugly. J Pathol 2009;217:282-298.

12. Katoonizadeh A, Nevens F, Verslype C, et al. Liver regeneration in acute severe liver impairment: a clinicopathological correlation study. Liver Int 2006;26:1225-1233.

13. Bird TG, Lorenzini $\mathrm{S}$, Forbes SJ. Activation of stem cells in hepatic diseases. Cell Tissue Res 2008;331:283-300.

14. Paku S, Nagy P, Kopper L, et al. 2-acetylaminofluorene dose-dependent differentiation of rat oval cells into hepatocytes: confocal and electron microscopic studies. Hepatology 2004;39:1353-1361.

15. Roskams TA, Libbrecht L, Desmet VJ. Progenitor cells in diseased human liver. Semin Liver Dis 2003;23:385-396.

16. Yovchev Ml, Grozdanov PN, Joseph B, et al. Novel hepatic progenitor cell surface markers in the adult rat liver. Hepatology 2007;45:139-149.

17. Yovchev $\mathrm{Ml}$, Grozdanov $\mathrm{PN}$, Zhou $\mathrm{H}$, et al. Identification of adult hepatic progenitor cells capable of repopulating injured rat liver. Hepatology 2008;47:636-647.

18. Gaudio E, Carpino G, Cardinale V, et al. New insights into liver stem cells. Dig Liver Dis 2009;41:455-462.

19. Lowes KN, Croager EJ, Olynyk JK, et al. Oval cell-mediated liver regeneration: Role of cytokines and growth factors. J Gastroenterol Hepatol 2003;18:4-12.

20. Dabeva MD, Shafritz DA. Activation, proliferation, and differentiation of progenitor cells into hepatocytes in the D-galactosamine model of liver regeneration. Am J Pathol 1993;143:1606-1620.

21. Laurent S, Horsmans $Y$, Starkel $P$, et al. Disrupted NF- kappa B activation after partial hepatectomy does not impair hepatocyte proliferation in rats. World J Gastroenterol 2005;11:7345-7350.
22. Van Hul NK, Abarca-Quinones J, Sempoux C, et al. Relation between liver progenitor cell expansion and extracellular matrix deposition in a CDE-induced murine model of chronic liver injury. Hepatology 2009;49:1625-1635.

23. Spee B, Carpino G, Schotanus BA, et al. Characterisation of the liver progenitor cell niche in liver diseases: potential involvement of Wnt and Notch signalling. Gut 2010;59:247-257.

24. Thenappan A, Li Y, Kitisin K, et al. Role of transforming growth factor beta signaling and expansion of progenitor cells in regenerating liver. Hepatology 2010;51:1373-1382.

25. Hu Z, Evarts RP, Fujio K, et al. Expression of hepatocyte growth factor and c-met genes during hepatic differentiation and liver development in the rat. Am J Pathol 1993;142:1823-1830.

26. Alison MR, Islam S, Lim SM. Number crunching in the cancer stem cell market. Breast Cancer Res 2009;11:302.

27. Dudas J, Mansuroglu T, Batusic D, et al. Thy-1 is an in vivo and in vitro marker of liver myofibroblasts. Cell Tissue Res 2007;329: 503-514.

28. Koenig S, Probst I, Becker $\mathrm{H}$, et al. Zonal hierarchy of differentiation markers and nestin expression during oval cell mediated rat liver regeneration. Histochem Cell Biol 2006;126:723-734.

29. Kon J, Ooe $\mathrm{H}$, Oshima $\mathrm{H}$, et al. Expression of CD44 in rat hepatic progenitor cells. J Hepatol 2006;45:90-98.

30. Roskams T. Liver stem cells and their implication in hepatocellular and cholangiocarcinoma. Oncogene 2006;25:3818-3822.

31. Evarts RP, Hu Z, Fujio K, et al. Activation of hepatic stem cell compartment in the rat: role of transforming growth factor alpha, hepatocyte growth factor, and acidic fibroblast growth factor in early proliferation. Cell Growth Differ 1993;4:555-561.

32. Sanchez A, Fabregat I. Growth factor- and cytokine-driven pathways governing liver stemness and differentiation. World J Gastroenterol 2010;16:5148-5161.

33. Harb R, Xie G, Lutzko C, et al. Bone marrow progenitor cells repair rat hepatic sinusoidal endothelial cells after liver injury. Gastroenterology 2009;137:704-712.

34. Nguyen LN, Furuya MH, Wolfraim LA, et al. Transforming growth factor-beta differentially regulates oval cell and hepatocyte proliferation. Hepatology 2007;45:31-41.

35. del Castillo G, Factor VM, Fernandez $M$, et al. Deletion of the Met tyrosine kinase in liver progenitor oval cells increases sensitivity to apoptosis in vitro. Am J Pathol 2008;172:1238-1247.

36. Watabe T, Miyazono K. Roles of TGF-beta family signaling in stem cell renewal and differentiation. Cell Res 2009;19:103-115.

37. Okaya A, Kitanaka J, Kitanaka $N$, et al. Oncostatin $M$ inhibits proliferation of rat oval cells, OC15-5, inducing differentiation into hepatocytes. Am J Pathol 2005;166:709-719. 